\title{
La seguridad en el paciente odontológico de acuerdo con la CONAMED.
}

\author{
Safety in the dental patient according to CONAMED.
}

\author{
José Eduardo Orellana-Centeno, ${ }^{*}$ Roxana Nayeli Guerrero Sotelo, ${ }^{\ddagger}$ Verónica Morales-Castillo ${ }^{\S}$
}

\section{RESUMEN}

La seguridad de los pacientes ha sido declarada por la Organización Mundial de la Salud (OMS) como un «principio fundamental para la atención sanitaria». Se menciona y define con la finalidad de caracterizar el problema de los incidentes relacionados con la atención de la salud, y especialmente sobre los eventos adversos. Existen definiciones básicas y operativas que sirven para elaborar reportes y proporcionar un marco referencial inicial, tal y como lo ha venido realizado en México la Comisión Nacional de Arbitraje Médico (CONAMED). La calidad en la atención es uno de los puntos más importantes, y se ha convertido poco a poco en un punto relevante en las agendas de los servicios de salud. Por esta razón debe afrontarse con un modelo de seguridad de los pacientes, con una visión que nos permita conocer los elementos básicos y conceptuales, así como la importancia de las medidas que el profesional en odontología da por hecho conocer por su entrenamiento dentro de las aulas universitarias.

Palabras clave: Seguridad, pacientes, odontología.

\section{ABSTRACT}

Patient safety has been declared by the World Health Organization (WHO) as a "fundamental principle for health care». It is mentioned and defined in order to characterize the problem of incidents related to health care, and especially about adverse events. There are basic and operational definitions that are used to prepare reports and provide an initial reference framework, as has been done in Mexico by the National Commission of Medical Arbitration (CONAMED). Quality of care is one of the most important points, gradually becoming a relevant point in the agendas of health services. It is the reason why it must be faced with a patient safety model, with a vision that allows us to know the basic and conceptual elements, as well as the importance of the measures that the professional in dentistry take for a fact to know for their Training within university classrooms.

Keywords: Safety, patients, dentistry.

\section{INTRODUCCIÓN}

$\mathrm{L}$ a seguridad de los pacientes ha sido declarada por la - Organización Mundial de la Salud (OMS) como un «principio fundamental para la atención sanitaria» desde 2002 cuando se estableció la Alianza Mundial para la

\footnotetext{
* Maestro en Salud Pública. Licenciatura de Odontología, Instituto de Investigación Sobre la Salud Pública, Universidad de la Sierra Sur, Oaxaca, México.

₹ Doctora en Procesos Políticos. Licenciatura de Enfermería, Instituto de Investigación Sobre la Salud Pública, Universidad de la Sierra Sur, Oaxaca, México.

§ Maestría en Administración. Unidad Académica Multidisciplinaria Zona Media, Universidad Autónoma de San Luis Potosí, San Luis Potosí, México.
}

Recibido: 03 Marzo 2020.

Aceptado para publicación: 30 Marzo 2020.
Seguridad del Paciente, y la cual ha sido definida como la atención sanitaria libre de daños asegurada por procesos de control, reducción o ausencia, para el paciente, del riesgo de daño innecesario, actual o potencial. Una finalidad de la seguridad de los pacientes es caracterizar los problemas de los incidentes relacionados a la atención de la salud, y especialmente los relativos a los eventos adversos y a los eventos centinelas. Existen definiciones básicas y operativas que sirven para elaborar reportes, quejas y proporcionar un marco referencial inicial. ${ }^{1}$ Para la OMS los eventos adversos pueden ser, por ejemplo, errores, desvíos o accidentes, en tanto que para el Sistema Nacional de Salud en México son cuasifallas, cuasiincidentes, daños (leves, moderados y severos), errores (médico por omisión y por comisión), entre otros. ${ }^{2}$

La seguridad de los pacientes se considera una nueva disciplina, en este sentido existen términos o conceptos ambiguos, vagos o contradictorios, lo cual puede conducir 
a problemas o confusiones al momento de clasificar un hecho o proporcionar un análisis y solución que ayude a los pacientes. Es por ello que algunas de las principales tareas son: a) el análisis y la ubicación de los conceptos para lograr un mejor resultado que sea de utilidad para la comunicación y la gestión organizacional, ${ }^{3}$ y b) la homologación y estandarización de los contenidos asignados a cada concepto o término.

\section{Principales estudios internacionales sobre eventos adversos}

To err is human: este reporte fue realizado por el Quality of Health Care in America Committee del Institute of Medicine de Estados Unidos, se basa en dos grandes estudios en Colorado-Utah y Nueva York, donde observaron $5.5 \%$ de eventos adversos en las hospitalizaciones, $10 \%$ conducía a la muerte y la mitad de los mismos eran evitables con prevención. Éste es uno de los estudios más importantes en el campo de la seguridad del paciente. ${ }^{1}$

ENEAS: es un estudio que se realizó en España (24 establecimientos hospitalarios) para determinar la incidencia de los eventos adversos, el cual detectó una incidencia de 9.3\% de los pacientes relacionados con la asistencia hospitalaria. Casi $22 \%$ de los eventos adversos fue el motivo de su reingreso, de éstos $16 \%$ eran graves. Dentro de las causas de dicha situación destacan: medicación (37\%), problemas técnicos del procedimiento (25\%), infecciones nosocomiales (25\%). Los eventos adversos en su mayoría se consideraron evitables (42\%). ${ }^{4}$

IBEAS: éste fue un estudio cooperativo internacional sobre la prevalencia de los efectos adversos en Latinoamérica, incluyó cinco países (México, Perú, Argentina, Costa Rica y Colombia) e involucró a 58 centros hospitalarios (11,555 pacientes). La prevalencia de los efectos adversos fue de $10.5 \%$. Se detectó que algunos de los factores que los provocaron fueron infección nosocomial $(37 \%)$, procedimiento $(28 \%)$, por cuidados $(13 \%)$, uso de medicamentos (8\%), diagnóstico (6\%). A consecuencia de los efectos adversos el tiempo de hospitalización aumentó con una media de 16 días y cerca de 18\% causaron reingreso. El porcentaje de eventos adversos que pudieron ser evitables es de $60 \%{ }^{2}$

\section{Terminología o conceptos}

En México la Dirección de Seguridad del Paciente, perteneciente a la Dirección General de Calidad y Educación en Salud emitió el glosario de términos aplicados a segu- ridad del paciente. ${ }^{3}$ Por su parte la Comisión Nacional de Arbitraje Médico CONAMED fue designada por la OMSOPS como centro colaborador en materia de calidad de la atención y la seguridad del paciente; en dicha materia ha emitido tanto los lineamientos generales para el cuidado de la seguridad del paciente como sus recomendaciones. En función de las directrices anteriores ofrecemos los siguientes conceptos fundamentales:

Cuasifalla: falta o error que no ocurrió. ${ }^{3}$

Cuasiincidente: «Evento que casi ocurrió o que ocurrió, pero del que nadie tiene conocimiento. Evento que podría haber tenido consecuencias no deseadas, pero no las tuvo porque no alcanzó al paciente, ya fuera por el azar o por una intervención oportuna.» ${ }^{3}$

Daño: alteración estructural o funcional del organismo y/o cualquier efecto perjudicial derivado de aquélla, y se clasifica en: leve que implica un daño mínimo y se equipara a un evento adverso; moderado que conlleva un daño significativo, pero no permanente y también se equipara al evento adverso; y finalmente severo que significa un daño permanente y se equipara a un evento centinela. ${ }^{3}$

Evento centinela: «Hecho inesperado que involucra la muerte o daño físico o psicológico grave y que no está relacionado con la historia natural de la enfermedad.» $\rangle^{3}$

Error: conducta clínica equivocada en la práctica médica o por cualquier profesional de la salud como consecuencia de la decisión de aplicar un criterio incorrecto, pudiendo ser por comisión al realizar una acción, o por omisión al dejar de realizarla. ${ }^{3}$ En tanto que la CONAMED establece que es una falla en el proceso de completar una acción tal como fue planeada o el uso de un plan equivocado para lograr un objetivo. No siempre provocan daño (incidentes si no provocan daño y si lo provocan se conocen como efecto adverso) o lesión. ${ }^{5}$

Cuasierror: es un incidente que no alcanza al paciente, pero que evidentemente debe ser considerado. ${ }^{6}$

Complicación: «Trastorno del paciente que surge durante el proceso de atención a la salud, sea cual fuere el entorno en que se dispensa. Enfermedad o lesión que surge a raíz de otra enfermedad y/o intervención asistencial.» ${ }^{3}$ En tanto que la CONAMED establece que es un resultado clínico adverso que de acuerdo con su magnitud puede provocar desenlaces como son: fallecimiento, lesiones graves (físicas y psicológicas). Requiere tratamiento que puede ser permanente o cambio en el estilo de vida que debería considerarse como una señal para cualquier sistema de vigilancia de la calidad en salud, aunque no todos los eventos adversos son señal o eventos 
centinelas, todos los eventos centinelas o señales sí son eventos adversos. ${ }^{7}$

Incidente sin daño: incidente que no llegó a causar daño, se puede llamar cuasifalla. Suceso aleatorio, imprevisto e inesperado que no produce daño al paciente ni pérdidas materiales o de cualquier tipo. ${ }^{3}$ En tanto que la CONAMED establece que es un incidente que ocurre en el proceso de atención de la salud que pudo afectar al paciente, pero que no existe la evidencia de que le haya causado daño alguno. Para algunos autores es ambiguo este término y consideran que es más conveniente usar el término incidente. Es comprensible porque el concepto sin daño en salud se asocia al término incidental o incidente. ${ }^{8}$

Evento adverso: «Incidente que produce daño leve o moderado al paciente. $\rangle^{3}$ En tanto que la CONAMED establece que es todo incidente imprevisto e inesperado como consecuencia de la atención (mayoritariamente) hospitalaria que produce la muerte, una discapacidad, la prolongación de estancia, o el reingreso subsecuente. ${ }^{9}$

Incidente: evento o circunstancia que ha ocasionado o podría haber ocasionado un daño al paciente, pero que no lo ha hecho. En este caso se podría omitir que se ocasionó un daño porque éste se asocia a un evento adverso. ${ }^{10}$

Infracción a las normativas: cuando el profesional de la salud desvía de forma deliberada las conductas o procesos que están definidos en las normas oficiales, guías de práctica clínica, manuales de procedimiento o protocolos clínicos. También se le conoce en el ámbito médico legal como conducta negligente. ${ }^{11}$

\section{Recomendaciones dictadas por la CONAMED}

A efecto de controlar, reducir o anular dichos eventos adversos la CONAMED ha emitido lineamientos y recomendaciones, entre las que destacan las siguientes: ${ }^{12}$

1. Identificación del paciente: generar un expediente clínico por cada paciente y mantener actualizada la información.

2. Comunicación efectiva: informar al paciente y en su caso a los familiares del tratamiento que vamos a realizar, que sea adecuadamente recibido, entendido y confirmado con la firma de la carta de consentimiento informado. Se recomienda no usar tecnicismos y en caso de ser necesarios explicarlos.

3. Seguridad en el proceso de medicación: cuidados al administrar anestésicos locales y cumplir con los requerimientos mínimos de la receta médica.
Los requerimientos mínimos de la receta médica

- Nombre del médico u odontólogo

- Nombre de la institución que la expide

- Título o grado académico

- Número de cédula profesional

- Domicilio

- Nombre del establecimiento

- Fecha de expedición del medicamento

4. Seguridad en los procedimientos: brindar al paciente todas la barreras de protección que sean necesarias para su procedimiento dental (limitar la exposición innecesaria a radiación por rayos $\mathrm{X}$ independientemente del uso de barreras de protección, oculares en todo procedimiento clínico, ingesta o inhalación de algún objeto) y manejo del protocolo de emergencias (curso para manejo de emergencias, botiquín de emergencia y cumplir con la NOM-005-SSA3-2010).

5. Reducción del riesgo de infecciones asociadas con la atención de la salud: implementar procedimientos estandarizados para lavado de manos del odontólogo y personal asistente, así como en la limpieza, desinfección y esterilización del instrumental clínico y áreas de trabajo. Utilizar de preferencia material desechable y de ser así no reutilizar. Siempre mostrar a los pacientes que el instrumental ha sido esterilizado y los desechables son nuevos.

6. Reducción del riesgo de daño al paciente por causas de caídas.

7. Registro y análisis de eventos centinela, adversos y cuasifallas: utilizar el expediente clínico del paciente para realizarlo.

8. Cultura de seguridad: utilizar trípticos, pósters, avisos o cualquier otro medio para difundir la seguridad del paciente así como promover y capacitar al personal odontológico y asistente. ${ }^{13}$

Para finalizar es de destacar que la CONAMED emitió en 2018 el Boletín de Seguridad del Paciente en Odontología, en el que se indica una lista de incidentes para prevenir en la atención estomatológica: ${ }^{7}$

1. Fractura de mandíbula del paciente.

2. Extracción de diente equivocado.

3. Tratar al paciente equivocado.

4. Aplicación del medicamento anestésico incorrecto.

5. Lesión del ojo del paciente debido a la falta de uso de protección ocular adecuada. 
6. Olvido de cuerpos extraños en el paciente después de procedimientos quirúrgicos.

7. Inhalación de cuerpos extraños por el paciente.

8. No esterilizar los instrumentos.

9. Falta de registro de la historia de las alergias del paciente a la medicación.

10. Uso de material dental en un paciente con alergia conocida al material dental utilizado (látex).

11. Prescripción de un medicamento a un paciente con una alergia conocida.

12. Reutilización de artículos desechables.

13. Falta de evaluación de cáncer oral de las lesiones del paciente que no se curan después de dos semanas de tratamiento.

14. Falta de realización de exámenes de detección del cáncer oral durante los exámenes de rutina.

15. Prescripción de medicamentos incorrectos para niños.

\section{La importancia de los registro de eventos}

La Organización Mundial de la Salud (OMS) refiere que para emprender iniciativas de seguridad adecuadamente orientadas, es fundamental comprender los problemas y los factores que contribuyen a crearlos. Por eso exhorta a que se establezcan y perfeccionen los sistemas de notificación y aprendizaje sobre la seguridad del paciente. ${ }^{14}$

El sistema de notificación debe de ser obligatorio para proporcionar información centrada en los errores asociados con lesiones graves o la muerte.

La notificación del evento se considera como el principal medio por el que se identifican los efectos adversos, con el propósito de mejorar la atención otorgada a un paciente; identificar y corregir las fallas de los sistemas, prevenir la repetición de los eventos y crear una base de datos para mejorar la calidad y gestión de riesgos y contribuir a un entorno seguro para la atención de los pacientes. ${ }^{15}$

\section{CONCLUSIONES}

La calidad en la atención es uno de los puntos más importantes, y se ha convertido poco a poco en un punto relevante en las agendas de los servicios de salud. Por esta razón debe afrontarse con un modelo de seguridad de los pacientes, con una visión que nos permita conocer los elementos básicos y conceptuales, así como la importancia de las medidas que el profesional en odontología da por hecho conocer por su entrenamiento dentro de las aulas universitarias.

El desarrollo de la cultura de la seguridad en el paciente trae beneficios como reducir la recurrencia y gravedad de los incidentes y el daño físico y psicológico en los pacientes, lo que conducirá a disminuir las consecuencias sociales y costos financieros que pudieran implicar debido a reclamaciones. La seguridad del paciente es una obligación desde el punto de vista ético y legal.

\section{BIBLIOGRAFÍA}

1. Schimmel EM. The hazards of hospitalization. Ann Intern Med. 1964; 60: 100-110.

2. Resultados del estudio IBEAS. Ministerio de Salud y Protección Social. 2010. Disponible en: https://www.minsalud.gov.co/sites/.../ RESULTADOS\%20ESTUDIO\%20IBEAS.pdf

3. Dirección General de Calidad y Educación en Salud. Glosario de términos aplicados a seguridad de los pacientes. Disponible en: http://www.calidad.salud.gob.mx/site/calidad/docs/dspsp_00F.pdf

4. Aranaz JM Aibar C, Galán A, Limón R, Requena J, Álvarez EE et al. La asistencia sanitaria como factor de riesgo: los eventos adversos ligados a la práctica clínica". Gac Sanit. 2006; 20 (Supl 1): 41-47.

5. Aguirre Gas H, Campos Castolo M, Carrillo Jaimes A. Análisis crítico de las quejas CONAMED, 1996-2007. Rev CONAMED. 2008; 13: 5-16.

6. Aranaz JM, Aibar C, Gea MT, León MT. Los efectos adversos en la asistencia hospitalaria, una revisión crítica. Med Clínica. 2004; 123 (1): $21-25$.

7. González Hermoso F. Errores médicos o desviaciones en la práctica asistencial diaria. Cir Esp. 2001; 69: 591-603.

8. Reason J. Human error: models and management. BMJ. 2000; 320: 768-770.

9. Rodríguez-Herrera R, Losardo RJ, Binvignat O. La anatomía humana como disciplina indispensable en la seguridad de los pacientes. Int J Morphol. 2019; 37 (1): 241-250.

10. Thomas EJ, Petersen LA. Measuring errors and adverse events in health care. J Gen Intern Med. 2003; 18 (1): 61-67.

11. Aranaz JM. Sucesos adversos relacionados con el uso del medicamento: ¿qué podemos aprender? Med Clín (Barc). 2006; 126 (3): 97-98.

12. Seguridad del paciente CONAMED. Memorias del Simposio CONAMED, 2010 ("Seguridad del paciente"). México: SS; 2010.

13. Secretaría de Salud. Recomendaciones para la seguridad del paciente en odontología. México: CONAMED; 2008.

14. Rodríguez-Herrera R, Mora EV, Yamaguchi-Díaz LP. Nuestra experiencia respecto al estudio IBEAS, retos y perspectivas. Boletín CONAMED-OPS. 2018; 4 (20): 36-37.

15. Secretaría de Salud, Subsecretaría de Innovación y Calidad. Dirección de Calidad y Educación en Salud. Comisión Interinstitucional de Enfermería. Prevención de Caídas en Pacientes Hospitalizados. Evaluación de la Calidad de los Servicios de Enfermería. Tres nuevos indicadores para la prevención de infecciones y seguridad del paciente. México: Secretaría de Salud; 2006. Cap. 2; pp. 19-34.

\section{Correspondencia:}

\section{José Eduardo Orellana-Centeno}

Guillermo Mijangos s/n, esq. Av. Universidad,

70800, Miahuatlán de Porfirio Díaz, Oaxaca, México.

E-mail: orellana17@msn.com 